\title{
EVALUACIÓN DE DOS VARIEDADES DE FRÉJOL DURANTE TRES ÉPOCAS DE SIEMBRA BAJO SISTEMA DE CULTIVO ASOCIADO CON MAÍZZ
}

\author{
${ }^{\bullet}$ Luis Godoy Montiel ${ }^{1}$, Gorki Díaz Coronel ${ }^{1}$, Gregorio Vásconez Montúfar ${ }^{1}$, Edwin Defaz Defaz ${ }^{2}$ y Betty González Osorio ${ }^{1}$ \\ ${ }^{1}$ Unidad de Investigación Cientifica y Tecnológica, Universidad Técnica Estatal de Quevedo, $\mathrm{km} 7$ vía \\ Quevedo - El Empalme, C. P. 73. Mocache,Los Ríos, Ecuador. ${ }^{\circ}$ lugomont@yahoo.es \\ ${ }^{2}$ Facultad de Ciencias Agrarias, Universidad Técnica Estatal de Quevedo, km 1 1/2 vía a Santo Domingo \\ de los Tsáchilas, C. P. 73. Quevedo, Los Ríos, Ecuador
}

Resumen

$\mathrm{E}^{1}$ objetivo fue estudiar el desarrollo agronómico y rendimiento de dos variedades de fréjol (Phaseolus vulgaris L.) "Ecuavegetal-2" (EVG-2) y "Pata de paloma" asociado con maíz (Zea mays L.) "INIAP H-552" durante tres épocas de siembra: 16 y 8 días antes de la siembra del maíz (dasm) y sembrados simultáneamente; además, establecer la eficiencia del uso de la tierra (RET) e ingresos (REI) de la asociación respecto de los monocultivos. "Pata de paloma" mostró menor incidencia de enfermedades, mayor número de vainas por planta y número de semillas por vaina; el mayor peso de 100 semillas lo promedió "EVG-2". En cuanto a las épocas de siembra, se obtuvo mayor número de vainas a los 8 y 16 dasm. Para los componentes del rendimiento, el fréjol asociado alcanzó mejores promedios que sembrado en monocultivo. Mejores rendimientos $\left(\mathrm{kg} \mathrm{ha}^{-1}\right)$ se registraron a los 16 y 8 dasm tanto para "EVG-2" como para "Pata de paloma" sin diferenciarse estadísticamente. El maíz sembrado en asociación no fue afectado en su desarrollo ni rendimiento tanto por las variedades de fréjol como por las épocas de siembra. Al analizar la RET y la REI, el sistema asociado sembrado 16 y 8 dasm mostró mayor eficiencia; los mayores coeficientes RET se dieron al asociar "Pata de paloma" con "INIAP H-552" a 16 y 8 dasm, y los mayores coeficientes REI se registraron al asociar "EVG-2" con "INIAP H-552" a 8 y 16 dasm.

Palabras claves: Asociación de cultivos, épocas de siembra, fréjol-maíz, RET y REI
$\mathrm{T}$ he objective was to study agronomic development and yield of two bean varieties "Ecuavegetal-2" (EVG-2) and "Pata de paloma", under intercropped system with "INIAP H-552" hybrid corn through three seed intervals: 16 and 8 days before corn seeding (dbcs) and seeding of beans simultaneously with corn and also to measure productivity using the Land Equivalent Ratio (LER) and economic LER of the intercropped systems along with single crops. "Pata de paloma" showed less incidence of diseases, higher number of legumes and number of seeds per plant, although better weight of 100 grains was for "EVG-2". About seeding dates, better number of legumes was obtained at 8 and $16 \mathrm{dbcs}$. Intercropped bean recorded better yield components averages than single crops. The yield $\left(\mathrm{kg} \mathrm{ha}^{-1}\right)$ at 16 and $8 \mathrm{dbcs}$, did not present significant differences. Intercropped corn was not affected either by bean varieties or seeding dates. LER and economic LER analyses show higher coefficients for intercropped systems especially when bean was planted at 16 y 8 dbcs, higher LER coefficients were found when "Pata de paloma" was intercropped with "INIAP H-552" at 16 and $8 \mathrm{dbcs}$ and higher REI coefficients were registered when "EVG-2" was intercropped with "INIAP H-552" at 8 and 16 dbcs.

Key words: Intercrop system, seeding dates, bean-corn, LER and economic LER

\section{INTRODUCCIÓN}

$E^{n}$ n el Ecuador existen zonas aptas para el normal desarrollo del cultivo del fréjol (P. vulgaris L.) como Milagro, Naranjito y Pedro Carbo en la provincia del Guayas; Babahoyo, Vinces y Quevedo en la provincia de Los Ríos. El área sembrada a nivel nacional es de

Recibido: 30-Noviembre-2010. Recibido en forma corregida: 18-Mayo2011. Aceptado: 24-Junio-2011.

Publicado como NOTA TÉCNICA en Ciencia y Tecnología 4(1): 5-11. 2011
60,000 ha con un rendimiento promedio de $550 \mathrm{~kg} \mathrm{ha}^{-1}$. La mayoría de la superficie sembrada es producto de las parcelas de pequeños agricultores (Robles, 2003). Los factores limitantes para la producción de fréjol se deben a la falta de variedades mejoradas y a la deficiente tecnología para el manejo del cultivo (SICA, 2004). Se denomina asociación de cultivos a la utilización de dos o más especies diferentes en forma simultánea y sirve para una mejor utilización del terreno, menor 
riesgo de pérdida por plagas y enfermedades en una de las especies (CIDICCO, 2004). El fréjol con el maíz es la forma de asociación más común entre los pequeños agricultores. Por lo general cuando el fréjol se siembra bajo este sistema, se lo hace durante los meses de mayo a junio y de forma simultánea (Robles, 2003). Se ha definido a las épocas de siembra de los cultivos asociados como lo días transcurridos a partir de la siembra de un cultivo con respecto a otro, tratando con ello de buscar un equilibrio en el crecimiento de ambos cultivos. Se habla de equilibrio, porque pueden haber situaciones de competencia, ya sea por luz, agua y nutrientes, así como de complementación, como es el caso del soporte prestado por un cultivo a otro; el concepto fundamental es minimizar la competencia y maximizar la complementación y/o transferencia entre ellos (Flor y Fracis, 1975). Sobre esta base, la investigación de la mejor época de siembra de los cultivares de fréjol tipo semiguía, "EVG-2" y "Pata de paloma" bajo el sistema de cultivo asociado, con relación al maíz, permitirá obtener la información necesaria para un mejor aprovechamiento de la tierra y un mejor rendimiento.

\section{Materiales y Métodos}

$\mathrm{L}$ a investigación se llevó a cabo durante los experimental "La María", de la Universidad Técnica Estatal de Quevedo localizada en el Km 7 de la vía Quevedo - El Empalme y ubicada geográficamente bajo las coordenadas de $79^{\circ} 27^{\prime}$ longitud oeste y $01^{\circ}$ 06' de latitud sur y a una altitud de $73 \mathrm{msnm}$. El clima de la zona es tropical húmedo, temperatura media anual de $25^{\circ} \mathrm{C}$, precipitación promedio anual de $2286.6 \mathrm{~mm}$, con humedad relativa media de $84 \%$ y heliofanía de 894 horas luz año ${ }^{-1}$. Presenta una topografía de relieve irregular y suelo con un $\mathrm{pH}$ promedio de 6.5 - 7.5.

\section{Material genético utilizado}

Las variedades de fréjol utilizadas fueron: "Ecuavegetal 2" (EVG-2) y "Pata de Paloma" (PP) ambas de tipo semiguía, además el híbrido de maíz "INIAP H-552".

\section{Diseño experimental y niveles de eficiencia}

Se utilizó un diseño de bloques completos al azar en arreglo factorial dos (variedades) $\mathrm{x}$ tres (épocas) + tres testigos (dos monocultivos de fréjol y uno de maíz) con cuatro repeticiones. Para la comparación de las medias de tipos de cultivo y épocas de siembra se utilizó la prueba de Tukey al 5\% de probabilidad en el error. Además se realizaron contrastes ortogonales entre grupos de tratamientos.

Para conocer los niveles de eficiencia de los sistemas asociados frente a los monocultivos, en relación a la producción y los ingresos netos, se aplicó: Razón de Equivalentes de la Tierra (RET) y Razón de Equivalentes de Ingresos (REI), de acuerdo a la metodología citada por Guamán Jiménez (1981):

\section{RET $=$ RMA/RMS + RFA/RFS}

\section{[1]}

Donde:
RET = Razón de Equivalentes de la Tierra
RMA $=$ Rendimiento del maíz asociado
RMS $=$ Rendimiento de maíz solo
RFA $=$ Rendimiento del fréjol asociado
RFS = Rendimiento del fréjol solo

\section{REI $=$ INMA/INMS + INFA/INFS}

\section{[2]}

Donde:

$$
\begin{aligned}
& \text { REI }=\text { Razón de Equivalentes de los Ingresos } \\
& \text { INMA }=\text { Ingresos netos del maíz asociado } \\
& \text { INMS }=\text { Ingresos netos del maíz solo } \\
& \text { INFA }=\text { Ingresos netos del fréjol asociado } \\
& \text { INFS }=\text { Ingresos netos del fréjol solo }
\end{aligned}
$$

La RET y/o la REI pueden ser igual a uno $(=1)$ lo cual indica una misma eficiencia en términos de uso de la tierra y de ingresos netos por hectárea de cultivo asociado que en el monocultivo; mayor a uno $(>1)$ indicando una mayor eficiencia en términos de producción y/o ingresos netos de los cultivos alternados que en el monocultivo y menor a uno $(<1)$ demostrando mejor eficiencia en términos de producción y/o ingresos netos de cualquiera de los monocultivos.

\section{Tratamientos y especificaciones de siembra}

Las variedades de fréjol, en asociación con el híbrido de maíz, se sembraron en tres épocas diferentes. El fréjol asociado fue comparado con el fréjol en monocultivo durante las tres épocas de siembra utilizadas a fin de conocer el efecto de la siembra asociada con la no asociada. Igual situación con el maíz. Los tratamientos fueron el resultado de las combinaciones entre los factores y los niveles en estudio (Variedades de fréjol x Épocas de siembra).

Se preparó el terreno con un pase de arada y dos de rastra; se efectuó la siembra con espeque depositando dos semillas por sitio; el raleo en el fréjol y el maíz se 
realizó a los ocho días dejando una planta por sitio; la fertilización fue fraccionada en tres partes para todos los cultivos, a razón de $80.0 \mathrm{~kg} \mathrm{ha}^{-1}$ de $\mathrm{N}$ (Urea), $40.0 \mathrm{~kg}$ $\mathrm{ha}^{-1}$ de $\mathrm{P}$ (Súper fosfato triple) y $60.0 \mathrm{~kg} \mathrm{ha}^{-1} \mathrm{de} \mathrm{K}$; para el control pre emergente de malezas se utilizaron los herbicidas Gramoxone (paraquat) y Prowl(pendimetalin) en dosis de 2.0 y $2.5 \mathrm{~L}$ por hectárea respectivamente junto a deshierbas manuales; para el control fitosanitario se aplicó $0.23 \mathrm{~kg} \mathrm{ha}^{-1}$ de Benex (benomil) más $0.56 \mathrm{~L}$ $\mathrm{ha}^{-1}$ de Attack (metamydofos). Para el control del hongo Thanatephorus cucumeris (Frank) Donk (estado sexual); Rhizoctonia solani Kuhn (estado asexual) causante de la enfermedad conocida como mustia hilachosa, se realizó una segunda aplicación utilizando $0.23 \mathrm{~kg} \mathrm{ha}^{-1}$ de Benex (benomil) y para el control de la mariquita (Cerotoma sp. y Diabrotica sp.) se aplicó Methavín (metomil) en dosis de $0.23 \mathrm{~kg} \mathrm{ha}^{-1}$.

Fue necesaria una tercera aplicación para el control de la mustia hilachosa y mariquitas, mezclando $0.23 \mathrm{~L} \mathrm{ha}^{-1}$ de Bavistin (carbendazin) más $0.56 \mathrm{~L} \mathrm{ha}^{-1}$ de Attack (metamydofos). Para el control del gusano cogollero del maíz (Spodoptera frugiperda) se aplicó insecticida Lorsban (clorpyrifos) a los veinte días, en dosis de 1.0 $\mathrm{L} \mathrm{ha}^{-1}$.

Adicionalmente, a los 40 días se preparó un "cebo" utilizando $50.0 \mathrm{~cm}^{3}$ de Lorsban (clorpyrifos) diluidos en dos litros de agua y mezclados en $10.0 \mathrm{~kg}$ de arena fina. Se aplicaron $4.0 \mathrm{~g} \mathrm{de}$ "cebo" aproximadamente en el ápice de cada planta; y finalmente la cosecha fue manual tanto para el fréjol, antes de que este se secara por completo, como para el maíz, a los 120 días de siembra.

\section{Mediciones experimentales}

En el fréjol se registraron los siguientes datos: Altura de planta, incidencia de mustia hilachosa utilizando la Guía de Evaluación de enfermedades del CIAT (1987), número de vainas por planta, número de semillas por vaina, peso de 100 semillas, rendimiento por planta y rendimiento por hectárea. Mientras que en el maíz se registró: Altura de planta, altura de inserción de mazorca, peso de 100 semillas, longitud de mazorca, peso de granos por mazorca, relación grano-tuza u olote obtenido de la división del peso de los granos para el peso de la tuza u olote y rendimiento por hectárea

\section{Resultados y Discusión}

\section{Fréjol}

Al analizar la altura de planta no se encontraron diferencias estadísticas $(\mathrm{P}>0.05)$ tanto para las variedades como para las épocas de siembra (Cuadro 1) lo que indicaría que las variedades de fréjol no se vieron afectadas en su desarrollo por las épocas en las que fueron sembradas con relación al maíz. En cuanto a la incidencia de mustia hilachosa, el menor porcentaje lo obtuvo la variedad "Pata de paloma" mientras en las épocas de siembra (Cuadro 1), no se encontraron diferencias estadísticas ( $\mathrm{P}>0.05)$ lo que coincide con lo publicado por Gálvez et al. (1989) que definen a esta enfermedad como típica de regiones cálidas y húmedas e independientemente si la siembra del fréjol se realiza en asociación o en monocultivo.
Cuadro 1. Efectos simples de dos variedades y tres épocas de siembra en la altura de planta y la incidencia de mustia hilachosa en el cultivo de fréjol asociado con el cultivo de maíz

\begin{tabular}{lcc}
\hline $\begin{array}{c}\text { Variedades y } \\
\text { Épocas }\end{array}$ & $\begin{array}{c}\text { Altura de } \\
\text { planta } \\
(\mathbf{c m})^{\dagger}\end{array}$ & $\begin{array}{c}\text { Incidencia } \\
\text { Mustia hilachosa }\end{array}$ \\
\hline Variedades & \\
\hline EVG - & & \\
Pata de Paloma & $18.78 \mathrm{a}$ & $21.25 \quad \mathrm{~b}$ \\
Épocas de siembra & & \\
16 dasm & $18.35 \mathrm{a}$ & $29.37 \mathrm{a}$ \\
8 dasm & $20.52 \mathrm{a}$ & $26.25 \mathrm{a}$ \\
Simultánea & $19.97 \mathrm{a}$ & $30.00 \mathrm{a}$ \\
\hline $\bar{X}$ & 19.61 & 28.54 \\
CV (\%) & 16.17 & 13.53 \\
\hline
\end{tabular}

${ }^{\dagger}$ Promedios con letras diferentes presentan diferencias de acuerdo a la prueba de Tukey $(\mathrm{P} \leq 0.05)$.

Al comparar el fréjol tanto en cultivo asociado como en monocultivo (Cuadro 2), no se encontraron diferencias estadísticas $(\mathrm{P}>0.05)$ para la altura de planta lo que demostraría un comportamiento estable del fréjol al sembrarse de forma asociada o en monocultivo mientras que el monocultivo de la variedad "Pata de paloma" promedió la menor incidencia de mustia hilachosa, resultados que coinciden con lo publicado por Simberloff (2000) para quien la incidencia de enfermedades fungosas, entre ellas la mustia hilachosa, se da mayormente en sistemas diversificados como es el caso de los cultivos asociados. 
Cuadro 2. Contrastes ortogonales de grupos de tratamientos del fréjol sembrado asociado con maíz y en monocultivo en la altura de planta y la incidencia de mustia hilachosa.

\begin{tabular}{|c|c|c|}
\hline Contrastes & $\begin{array}{l}\text { Altura de } \\
\text { planta } \\
(\mathrm{cm})^{\dagger}\end{array}$ & $\begin{array}{c}\text { Incidencia } \\
\text { Mustia } \\
\text { hilachosa } \\
(\%) \dagger\end{array}$ \\
\hline $\begin{array}{l}\text { Asociación } \\
\text { vs }\end{array}$ & $19.6 \mathrm{a}$ & $28.5 \mathrm{a}$ \\
\hline Monocultivo & $18.9 \mathrm{a}$ & $26.3 \mathrm{a}$ \\
\hline $\begin{array}{l}\text { Monocultivo EVG-2 } \\
\text { vs }\end{array}$ & $18.5 \mathrm{a}$ & $35.0 \mathrm{a}$ \\
\hline Monocultivo PP & $19.3 \mathrm{a}$ & $17.5 \mathrm{~b}$ \\
\hline
\end{tabular}

Al analizar los componentes del rendimiento (Cuadro 3), la variedad Pata de Paloma alcanzó los mayores promedios tanto para el número de vainas por planta, que podría deberse a su tradicional utilización como cultivo asociado con maíz y la consecuente adaptación a las condiciones ambientales y agronómicas de esta zona, así como para el número de semillas por vaina cuyos promedios están contenidos dentro de los parámetros presentados por Erazo (2005) quien cuantifica entre cuatro y seis semillas por vaina a la variedad "Pata de paloma". Para el peso de cien semillas, la variedad "EVG-2" alcanzó el mayor peso promedio (Cuadro 3) coincidiendo nuevamente con Erazo (2005) quien cita un peso promedio de entre 20 a $23 \mathrm{~g}$ de esta variedad para dicha variable. En cuanto al rendimiento por planta, no se encontraron diferencias estadísticas $(\mathrm{P}>0.05)$ entre las variedades.

Para las épocas de siembra, el mayor número de vainas por planta y de rendimiento por planta (Cuadro 3) se alcanzó a los 16 días antes de la siembra del maíz (dasm) lo que difiere de lo expuesto por Francis et al. (1982) quienes luego de estudiar la influencia de las épocas de siembra sobre la competencia y el rendimiento en cultivos asociados, concluyeron que la competencia del maíz hacia el fréjol reduce los componentes del rendimiento de este último en detrimento de si la siembra se adelanta o no. Mientras que para el número de semillas por vaina y peso de cien semillas (Cuadro 3), los menores promedios se encontraron cuando se sembró el fréjol simultáneamente con el maíz y los mayores para las siembras realizadas 16 y 8 dasm aunque sin mostrar diferencias estadísticas $(\mathrm{P}>0.05)$ entre ellas lo que podría relacionarse con el óptimo desarrollo alcanzado por el fréjol al no tener ninguna competencia por nutrientes, espacio, agua y luz debido a los días transcurridos entre la siembra de éste y del maíz.

Cuadro 3. Efectos simples de dos variedades y tres épocas de siembra para cuatro componentes del rendimiento en el cultivo de fréjol sembrado en asociación con el cultivo de maíz

\begin{tabular}{lcccc}
\hline Variedades y Épocas & $\begin{array}{c}\text { Vainas } \\
\text { por planta }^{\dagger}\end{array}$ & $\begin{array}{c}\text { Semillas } \\
\text { por vainas }^{\dagger}\end{array}$ & $\begin{array}{c}\text { Peso 100 } \\
\text { semillas (g) } \dagger\end{array}$ & $\begin{array}{c}\text { Rendimiento } \\
\text { por planta (g) }\end{array}$ \\
\hline Variedades & & & & \\
$\quad$ EVG -2 & $13.59 \mathrm{~b}$ & $4.57 \mathrm{~b}$ & $23.40 \mathrm{a}$ & $14.83 \mathrm{a}$ \\
$\quad$ Pata de Paloma & $15.97 \mathrm{a}$ & $5.05 \mathrm{a}$ & $19.40 \mathrm{~b}$ & $15.55 \mathrm{a}$ \\
Épocas de siembra & & & & \\
$\quad 16$ dasm & $18.06 \mathrm{a}$ & $5.09 \mathrm{a}$ & $22.40 \mathrm{a}$ & $20.40 \mathrm{a}$ \\
8 dasm & $16.04 \mathrm{~b}$ & $4.97 \mathrm{a}$ & $22.30 \mathrm{a}$ & $16.66 \quad \mathrm{~b}$ \\
Simultánea & $10.24 \quad \mathrm{c}$ & $4.36 \mathrm{~b}$ & $19.60 \mathrm{~b}$ & $8.51 \quad \mathrm{c}$ \\
\hline $\bar{X}$ & 14.78 & 4.81 & 21.42 & 15.19 \\
CV (\%) & 9.83 & 5.10 & 6.94 & 14.42 \\
\hline
\end{tabular}

${ }^{\dagger}$ Promedios con letras diferentes presentan diferencias estadísticas, de acuerdo a la prueba de Tukey $(\mathrm{P} \leq 0.05)$. 
Cuadro 4. Contrastes ortogonales de grupos de tratamientos del fréjol sembrado en asociación con maíz y en monocultivo para el número de vainas, semillas por vaina, peso de 100 semillas y rendimiento por planta

\begin{tabular}{lcccc}
\hline \multicolumn{1}{c}{ Contrastes } & $\begin{array}{c}\text { Vainas } \\
\text { por planta } \uparrow\end{array}$ & $\begin{array}{c}\text { Semillas } \\
\text { por vainas } \dagger\end{array}$ & $\begin{array}{c}\text { Peso 100 } \\
\text { semillas (g) } \dagger\end{array}$ & $\begin{array}{c}\text { Rendimiento } \\
\text { por planta (g) }\end{array}$ \\
\hline $\begin{array}{l}\text { Asociación } \\
\text { vs }\end{array}$ & $14.8 \mathrm{a}$ & $4.8 \mathrm{a}$ & $21.4 \mathrm{a}$ & $15.2 \mathrm{a}$ \\
Monocultivo & $8.8 \mathrm{~b}$ & $3.9 \mathrm{~b}$ & $17.1 \quad \mathrm{~b}$ & $5.7 \mathrm{~b}$ \\
$\begin{array}{l}\text { Monocultivo EVG-2 } \\
\text { vs }\end{array}$ & $8.9 \mathrm{a}$ & $4.0 \mathrm{a}$ & $18.5 \mathrm{a}$ & $6.4 \mathrm{a}$ \\
Monocultivo PP & $8.7 \mathrm{a}$ & $3.9 \mathrm{a}$ & $15.7 \quad \mathrm{~b}$ & $5.1 \mathrm{a}$ \\
\hline
\end{tabular}

${ }^{\dagger}$ Promedios con letras diferentes presentan diferencias estadísticas, de acuerdo a la prueba de Tukey $(\mathrm{P} \leq 0.05)$.

$\mathrm{Al}$ agrupar los tratamientos y compararlos entre ellos para analizar los componentes del rendimiento del fréjol (Cuadro 4), los mejores promedios se encontraron cuando se sembró el fréjol en asociación con maíz lo que difiere, para el caso del número de vainas por planta, de lo publicado por Marín y Cavanerio (1998) respecto a un ensayo de rendimiento en granos bajo sistema de cultivo asociado fréjol-maíz donde encontraron un menor número de legumbres o vainas en comparación con los monocultivos debido a la competencia del fréjol con el maíz; el promedio obtenido para el número de semillas por vaina es diferente de lo publicado por Arias y Muñoz (1983) para quienes el número de semillas por vaina es mayor en los monocultivos; el peso de cien semillas también difiere a lo publicado por Aguilar (1976) donde manifiesta haber encontrado un mayor peso de cien semillas en los monocultivos y en cuanto al rendimiento por planta, este se relaciona con la importancia de las plantas de maíz como tutores para las guías de las plantas de fréjol a diferencia de los monocultivos de fréjol donde no se observó un buen desarrollo de sus guías que se volvieron rastreras conteniendo menos vainas y semillas por vainas, debido a la alta incidencia de enfermedades y pérdida de granos según lo publicado por Flor y Francis (1975).

Con respecto al rendimiento por hectárea, no se encontraron diferencias $(\mathrm{P}>0.05)$ al analizar las variedades lo que indica que estas no se vieron afectadas ni por las épocas ni por los sistemas de siembra estudiados. Al analizar las épocas de siembra, el menor promedio se obtuvo al sembrar fréjol de forma simultánea con maíz (Cuadro 5) coincidiendo con lo enunciado por Prager (1977) para quien entre más se adelante la siembra de fréjol a la del maíz, más altas producciones se conseguirán en éste y con siembras iguales o posteriores, los rendimientos del fréjol disminuyen significativamente. Los promedios más altos se dieron al adelantar la siembra del fréjol en 16 y 8 dasm pero sin mostrar diferencias $(\mathrm{P}>0.05)$ coincidiendo con lo publicado por la Universidad Nacional Agraria (1976) sobre un estudio donde concluyeron que una ventaja de quince días en la siembra del fréjol sobre el maíz logra rendimientos más altos (Cuadro 5).

Cuadro 5. Efectos simples de dos variedades y tres épocas de siembra para el rendimiento por hectárea en el cultivo de fréjol sembrado en asociación con el cultivo de maíz

\begin{tabular}{lc}
\hline Variedades y Épocas & $\begin{array}{c}\text { Rendimiento } \\
\left(\mathbf{k g ~ h a}^{-1}\right)^{\dagger}\end{array}$ \\
\hline Variedades & $462.3 \mathrm{a}$ \\
EVG - 2 & $435.3 \mathrm{a}$ \\
Pata de Paloma & \\
Épocas de siembra & $577.4 \mathrm{a}$ \\
16 dasm & $510.8 \mathrm{a}$ \\
8 dasm & $204.1 \quad \mathrm{~b}$ \\
Simultánea & 441.6 \\
\hline $\bar{X}$ & 15.21 \\
\hline
\end{tabular}

${ }^{\dagger}$ Promedios con letras diferentes presentan diferencias estadísticas, de acuerdo a la prueba de Tukey $(\mathrm{P} \leq 0.05)$.

Maíz

Al analizar las variables altura de planta y altura de inserción de la mazorca en el maíz, no se 
encontraron diferencias $(\mathrm{P}>0.05)$ cuando este se sembró asociado con las dos variedades de fréjol, durante las tres épocas de siembra bajo estudio y tampoco al comparar la asociación de éste con el fréjol y su respectivo monocultivo indicando poca o nula interferencia agronómica de las dos variedades de fréjol hacia el maíz durante su desarrollo (Marin y Cavanerio, 1998). Asimismo, para las variables peso de cien semillas, longitud de mazorca, peso de mazorca, peso de grano por mazorca y relación grano/tuza, no se encontraron diferencias estadísticas $(\mathrm{P}>0.05)$ al analizar las variedades, las épocas de siembra y al comparar la asociación frente al monocultivo.

En cuanto al rendimiento por hectárea, al analizar las variedades, no se obtuvieron diferencias ( $>$ > 0.05) entre ellas. Por otro lado los mejores rendimientos se obtuvieron cuando el maíz se sembró 8 y 16 días más tarde que el fréjol (Cuadro 6) aunque no se encontraron diferencias $(\mathrm{P}>0.05)$, concordando con la investigación realizada por el CIDICCO (2002) en Honduras, donde se indica que el fréjol no limita el desarrollo ni el rendimiento del maíz.

Cuadro 6. Efectos simples del maíz en asociación con dos variedades de fréjol en épocas de siembra para el rendimiento por hectárea

\begin{tabular}{lc}
\hline Variedades y Épocas & $\begin{array}{c}\text { Rendimiento } \\
\left(\mathbf{k g ~ h a}^{-1}\right)^{\dagger}\end{array}$ \\
\hline Variedades & $3177.6 \mathrm{a}$ \\
$\mathrm{EVG}-2$ & $3219.2 \mathrm{a}$ \\
Pata de Paloma & \\
Épocas de siembra & $3347.0 \mathrm{a}$ \\
16 dasm & $3353.7 \mathrm{a}$ \\
8 dasm & $2894.4 \quad \mathrm{~b}$ \\
\hline Simultánea & 3198.4 \\
\hline $\bar{X}$ & 10.21 \\
\hline
\end{tabular}

${ }^{\dagger}$ Promedios con letras diferentes presentan diferencias estadísticas, de acuerdo a la prueba de Tukey $(\mathrm{P} \leq 0.05)$.

\section{Análisis de la RET y la REI}

Al analizar la RET y REI (Cuadro 7), se determinó que el sistema de cultivo de fréjol asociado con maíz es más eficiente tanto en uso de la tierra como de los ingresos en comparación con los monocultivos, resultados similares a los reportados por Guamán Jiménez
(1981). En general, a los 16 y 8 dasm se reportaron los mayores coeficientes de la RET y REI confirmando la conveniencia de sembrar el fréjol con anticipación del maíz. La variedad criolla "Pata de paloma" alcanzó los mayores coeficientes RET, demostrando los mejores rendimientos por superficie cultivada de asociación de esta variedad con respecto a su monocultivo; la variedad "EVG-2" obtuvo los mayores coeficientes REI lo cual demuestra una mayor eficiencia de ingresos de esta variedad en asociación con maíz respecto de su monocultivo.

Cuadro 7. Razón de Equivalentes de la Tierra (RET) y del Ingreso (REI) de dos variedades de fréjol durante tres épocas de siembra en asociación con maíz

\begin{tabular}{lcccccc}
\hline & \multicolumn{3}{c}{ RET } & \multicolumn{3}{c}{ REI } \\
\cline { 2 - 7 } Asociación & $\begin{array}{c}\mathbf{1 6} \\
\text { dasm }\end{array}$ & $\begin{array}{c}\mathbf{8} \\
\text { dasm }\end{array}$ & $\begin{array}{c}\text { Simul- } \\
\text { tánea }\end{array}$ & $\begin{array}{c}\mathbf{1 6} \\
\text { dasm }\end{array}$ & $\begin{array}{c}\mathbf{8} \\
\text { dasm }\end{array}$ & $\begin{array}{c}\text { Simul- } \\
\text { tánea }\end{array}$ \\
\hline EVG 2 + & 2.3 & 2.2 & 1.4 & 1.8 & 2.5 & 2.1 \\
INIAP H-552 & & & & & & \\
\hline PP + & 2.5 & 2.3 & 1.3 & 1.6 & 2.1 & 1.4 \\
INIAP H-552 & & & & & & \\
\hline
\end{tabular}

\section{Conclusiones}

\section{Fréjol}

La variedad "Pata de paloma" mostró menor incidencia de mustia hilachosa, mayor número de vainas por planta y semillas por vaina; la variedad "EVG-2" promedió mayor peso de cien semillas; tanto "Pata de paloma" como "EVG-2" no reportaron diferencias estadísticas para el rendimiento por planta y rendimiento por hectárea. Las épocas de siembra fueron significativas para las variables número de vainas y el rendimiento por planta cuando se sembró el fréjol 16 dasm, los menores promedios se obtuvieron al sembrar el fréjol de forma simultánea al maíz en las variables número de semillas por vainas, peso de cien semillas y rendimiento por hectárea. La asociación de cultivos fue superior en las variables número de vainas por planta, número de semillas por vaina, peso de cien semillas y rendimiento por planta con mayores promedios que los alcanzados por los monocultivos. No se encontraron diferencias estadísticas para la altura de planta ni en las variedades ni en las épocas de siembra, tampoco al comparar los cultivos asociados con los monocultivos.

Maíz

El desarrollo del maíz y los componentes del rendimiento no fueron afectados al asociarlo con dos variedades de fréjol y establecerlo durante tres épocas 
de siembra. Los mejores rendimientos del maíz (kg ha-1) se obtuvieron cuando se lo sembró asociado con el fréjol a los 16 y 8 dasm aunque sin mostrar diferencias estadísticas entre ellos.

\section{Análisis de la RET y la REI}

Al analizar la RET y la REI se determinó una mayor eficiencia de la asociación respecto del monocultivo cuando la siembra del fréjol se realizó a los 16 y 8 dasm. Los mayores coeficientes RET se encontraron en la asociación de "Pata de paloma" con "INIAP H-552" a los 16 y 8 dasm. Mientras que los mayores coeficientes REI se encontraron en la asociación de "EVG-2" con "INIAP H-552" a los 8 y 16 dasm.

\section{LiterATURA CITADA}

Aguilar, M. 1976. Cultivo asociado maíz (Zea mays), fríjol chiclayo (Vigna sinensis) en la zona de Iquitos. Tesis Ing. Agrónomo, Programa Agronomía UNAP. Iquitos-Perú. 52 p.

Arias, A. y C. Muñoz, 1983. Evaluación de sistemas en monocultivo y asociación de maíz y leguminosas en el nor-oriente de Guárico, Perú. Agronomía Tropical. 33(1-6): 143-154.

CIDICCO (Centro Internacional de información sobre Cultivos de Cobertura) 2002. Boletín electrónico Noticias sobre cultivos de cobertura No. 12. (en línea). Consultado 23 Abr 2006. Disponible en http://www.cidicco.hn/archivospdf/Boletin12.pdf.

CIDICCO (Centro Internacional de información sobre Cultivos de Cobertura) 2004. Uso del fréjol Chinapopo. (en línea).Consultado 16 Agost 2006. Disponible en http://www.cidicco.hn/

CIAT (Centro Internacional de Agricultura Tropical). 1987. Sistema estándar para la evaluación de germoplasma de fríjol. Aart Van Schoonhover y Marcial A. Pastor-Corrales (comps.). Cali, Colombia. 56 p.

Erazo, W. 2005. Evaluación de once variedades de fréjol (Phaseolus vulgaris L.) durante la época seca del año 2004 en la zona de Quevedo. Tesis Ing. Agr. Universidad Técnica Estatal de Quevedo. Facultad de Ciencias Agrarias. Escuela de Ingeniería Agronómica. 57 p.

Flor, A. y C. Francis. 1975. Propuesta de estudio de algunos componentes de una metodología para investigar los cultivos asociados en el trópico latinoamericano. XXII Reunión de PCCMCA. San Salvador, Salvador. 37 p.
Francis, C.A.; M. Prager and G. Tejeda. 1982. Effects of relative planting dates in bean (Phaseolus vulgaris L.) and maize (Zea mays L.) intercropping patterns. Field Crops Research. No 5: 45-54.

Gálvez, G.; B. Mora, M.A. Pastor. 1989. Web blight. In: Pastor, M.; Schwartz, H. Bean production problems in the tropics. CIAT-Cali, Colombia. p. $195-209$.

Guamán R.W. 1981. Rendimiento físico y económico del fríjol (Phaseolus vulgaris L.) y maíz (Zea mays L.) sembrados solos y en asociación. Tesis de Maestría en Ciencias. Colegio de Postgraduados Chapingo, México. 122 p.

Marín, C. y R. Cavanerio. 1998. Crecimiento y rendimiento en granos en una asociación de maíz (Z. mays L.) y caraota (Phaseolus vulgaris L.), con siembra simultánea. Revista de la Facultad de Agronomía Universidad del Zulia. Maracaibo, Venezuela. No. 8: 27-32.

Prager, M. 1977. Factores agronómicos en la producción fríjol/maíz. Información de resultados de investigación. CIAT- Palmira. Colombia. 17 p.

Robles, C. 2003. Comportamiento agronómico de diez variedades de fréjol de exportación, en diferentes zonas del Litoral Ecuatoriano. Guayaquil, EC. (en línea). Consultado 14 Marz 2005. Disponible en: http:// www.sica.gov.ec/ agronegocios/ index. html.

SICA EC. 2004 (Servicio de Información y Censo Agropecuario). Fenología agrícola del fréjol. (en línea). Consultado 20 Dic 2005. Disponible en: http://www.sica.gov.ec/.

Simberloff, D. 2000. Amenaza a la biodiversidad y que se puede hacer. (en línea). Consultado 12 Oct 2005. Disponible en: http://www.actionbioscience.org/ esp/ biodiversity/simberloff.html.

UNIVERSIDAD NACIONAL AGRARIA. 1976. Informativo de maíz. Programa Cooperativo de Investigación en Maíz. Lima-Perú. 9 p. Boletín $\mathrm{N}^{\mathrm{o}} 14$ 